\title{
Analysis and Compensation of the Inner-arm Error in the North-finder Syetem of SINS
}

\author{
Lei Wang ${ }^{1}$, Zhou Wang ${ }^{2}$, Yuan Ren ${ }^{2}$ \\ ${ }^{1}$ Graduate School, Aerospace Engineering University, Beijing 101416, China; \\ ${ }^{2}$ Department of Space Equipment, Aerospace Engineering University, Beijing 101416, China
}

\begin{abstract}
For the high precision strapdown inertial navigation system in north, the rotary modulation will not get rid of the inner-arm error.In the north-seeking process, because of the rotating device, it will introduce the error into the north navigation calculation. In this paper, first, the error of the within arm is modeled and analyzed, and the mathematical expression of the error effect is deduced. And two factors affecting the error of the within arm are analyzed and determined, then error compensation method of the within arm is put forward. Finally the simulation results are verified.
\end{abstract}

\section{Introduction}

The IMU rotation is used to drive the IMU spin and the navigation solution is used in the conventional strap-homing system. According to the traditional error compensation method, the common error can be removed by rotation modulation ${ }^{[1]}$. In the course of the study, the emphasis should be placed on the error which can not be demodulated with the rotation modulation, or the research and analysis of some new errors introduced after the rotation device is added.

Because of the mechanical structure and the reason of the system, the three accelerometers in the IMU are not superimposed. The three accelerometers measure the ratio of each acceleration meter to the direction of its input axis. In the process of angular motion, the error of the accelerometer is bound to result in the error of finding the north navigation solution, which is commonly referred to as the inner-arm error (also known as the size effect) ${ }^{[2]}$. In the process of this study, using the gyroscope for high precision laser gyro, due to the physical structure of the optical does not exist in the mechanical rotor, is not sensitive to the change of the acceleration, therefore optical gyro output is not affected by the influence of size effect, there is no error of the lever arm. However, in the navigation solution calculation, the measurement error caused by the error effect of the inside lever arm of the accelerometer can not be ignored, which can affect the north navigation results.

\section{The analysis and modeling of inner-arm error}

If you look at the IMU as a rigid body, according to the rigid body theory, in the IMU motion, the angular velocity of each point on it is the same. So for laser gyro in the company, the installation position and orientation of three gyro can, as long as there is no parallel between three axis gyro sensitive relationship can, after calibration can achieve three-dimensional angular velocity measurement. But for accelerometer, due to the three accelerometer physical size and the limitation of the actual installation position, makes them measurement of acceleration is the direction of the IMU differences, if do not take this factor into consideration, the three accelerometer to measure the acceleration as the output of the "point measuring component" for north and navigation, so will lead to navigation errors. If there is a large internal lever error, the angular acceleration measured by the accelerometer will cause a certain error to the north seeking result, and the result will be affected. In inertial navigation system on the filter, if use speed as observed quantity, if the company is larger in the lever arm, in the case of the rotating modulation, plus the indexing mechanism of IMU continuously rotates, it will bring bigger error on observation, affect the alignment accuracy.

In the inertial measurement unit, the three accelerometers are installed in three direction positions on the northeast sky axis respectively, and the relative position remains constant. The extension cord of the three accelerometer's sensitive axis intersects at the origin $\mathrm{O}$ point of the coordinate system. The specific location installation is shown in figure 1: 

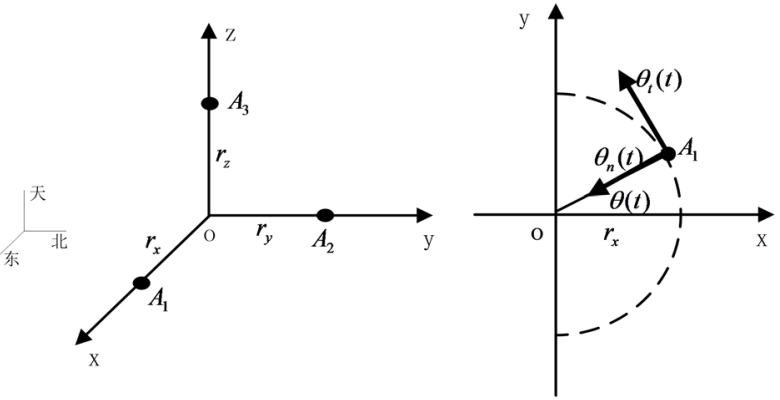

Figure 1. The installation schematic of accelerometer

Because the two vertical installations of the three accelerometers. In the process of analysis, an example is given in the $\mathrm{X}$-axis direction, and the partial error is used in the process of seeking north. Assuming that the IMU rotates around the $\mathrm{Z}$ axis, in the xy axis that forms the horizontal plane, assuming that the Angle between the accelerometer $\mathrm{A}$ and $\mathrm{Ox}$ is the sinusoidal Angle motion:

$$
\phi(t)=\phi_{0} \sin (\omega t)
$$

In the formula: the $\phi_{0}$ is to define the amplitude of vibration; $\omega$ is the rotation angular velocity; the oscillation period is $T=2 \pi / \omega$.

Taking the derivative of the upper formula, the angular velocity of rotation can be obtained:

$$
\dot{\phi}(t)=\phi_{0} \omega \cos (\omega t)
$$

According to formula (1) and formula (2), we can have the normal velocity, the centripetal acceleration, the tangent velocity and tangential acceleration of accelerometer A:

$$
\begin{aligned}
& v_{n}(t)=0 \\
& a_{n}(t)=r_{A}(\dot{\phi}(t))^{2}=r_{A} \phi_{0}^{2} \omega^{2} \cos ^{2}(\omega t) \\
& v_{t}(t)=r_{A} \dot{\phi}(t)=r_{A} \phi_{0} \omega \cos (\omega t) \\
& a_{t}(t)=\left(v_{t}(t)\right)^{\prime}=-r_{A} \phi_{0} \omega^{2} \cos (\omega t)
\end{aligned}
$$

In the formula (3), the velocity component can be obtained by decomposing the projection of the normal velocity and tangent velocity along the axis $x y z$ :

$$
\begin{aligned}
v_{x}(t) & =v_{n x}(t)+v_{t x}(t)=0-v_{t}(t) \sin [\phi(t)] \\
& =-r_{A} \phi_{0} \omega \cos (\omega t) \sin \left[\phi_{0} \cos (\omega t)\right] \\
v_{y}(t) & =v_{n y}(t)+v_{t y}(t)=0+v_{t}(t) \cos [\phi(t)] \\
& =r_{A} \phi_{0} \omega \cos (\omega t) \cos \left[\phi_{0} \cos (\omega t)\right]
\end{aligned}
$$

In formula (3), the acceleration projection can be obtained by decomposing the tangent acceleration and the normal acceleration along the axis:

According to the above formula (4), easy to know:

$$
v_{x}(0)=v_{x}(T)=o ; v_{y}(0)=v_{y}(T)=r \phi_{0} \omega
$$

The following relation can be obtained by taking the derivative of formula (4) :

$$
\begin{aligned}
& v_{x}(t)=v_{t x}(t)+v_{n x}(t) \\
& v_{y}(t)=v_{t y}(t)+v_{n y}(t)
\end{aligned}
$$

$$
\begin{aligned}
a_{n x}(t) & =-a_{n}(t) \cos [\phi(t)] \\
& =-r_{A} \phi^{2}{ }_{0} \omega^{2} \cos ^{2}(\omega t) \cos \left[\phi_{0} \sin (\omega t)\right] \\
a_{n y}(t) & =-a_{t}(t) \sin [\phi(t)] \\
& =-r_{A} \phi^{2}{ }_{0} \omega^{2} \cos ^{2}(\omega t) \sin \left[\phi_{0} \sin (\omega t)\right] \\
a_{t x}(t) & =-a_{t}(t) \sin [\phi(t)] \\
& =r_{A} \phi_{0} \omega^{2} \cos (\omega t) \sin \left[\phi_{0} \sin (\omega t)\right] \\
a_{t y}(t) & =a_{n}(t) \cos [\phi(t)] \\
& =-r_{A} \phi_{0} \omega^{2} \cos (\omega t) \cos \left[\phi_{0} \sin (\omega t)\right]
\end{aligned}
$$

Defining function:

$$
\begin{aligned}
& f_{1}\left(x, t_{1}, t_{2}\right)=\int_{t_{1}}^{t_{2}} x \sin t \sin (x \sin t) d t \\
& f_{2}\left(x, t_{1}, t_{2}\right)=\int_{t_{1}}^{t_{2}} x \sin t \cos (x \sin t) d t
\end{aligned}
$$

According to the symmetry and periodicity of the integrand, it can be obtained:

$$
\begin{aligned}
f_{1}(x, o, \pi / 2) & =f_{1}(x, \pi / 2, \pi)=f_{1}(x, \pi, 3 \pi / 2) \\
& =f_{1}(x, 3 \pi / 2,2 \pi) \\
f_{2}(x, o, 2 \pi) & =f_{2}(x, \pi / 2,3 \pi / 2)=0
\end{aligned}
$$

In one cycle, the acceleration integral along the coordinate axis can be obtained by the increment of velocity:

$$
\left\{\begin{array}{c}
\Delta v_{t x}(T)=\int_{o}^{T} a_{t x}(t) d t=4 r_{A} \omega f_{1}\left(\phi_{0}, 0, \pi / 2\right) \\
\Delta v_{t y}(T)=\int_{o}^{T} a_{t y}(t) d t=-f_{2}\left(\phi_{0}, 0,2 \pi\right)=0 \\
\Delta v_{n x}(T)=\int_{o}^{T} a_{n x}(t) d t=-4 r_{A} \omega f_{1}\left(\phi_{0}, 0, \pi / 2\right) \\
\Delta v_{n y}(T)=\int_{o}^{T} a_{n y}(t) d t=0
\end{array}\right.
$$

\section{Error analysis of the inner-arm effect}

In the rotation process, the three accelerometers are assumed to be perpendicular to each other and the sensitive axis intersects at point $o_{b}$. Assume that the rotation axis and the rotation axis are not at the same point, so the axis of rotation is perpendicular to $o_{b} z_{b}$, the rotation angular velocity is $\omega$, and the amplitude of vibration is $\phi_{0}$.

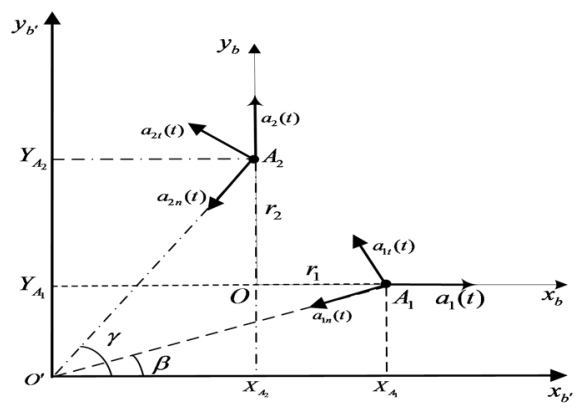

Figure 2. Schematic diagram of installation in the xy plane 
The measured value of the accelerometer is the sum of the projection of the centripetal acceleration and tangent acceleration at a point in the direction of the accelerometer.

The centripetal acceleration andtangential acceleration at the accelerometer of $A_{1}$ can be expressed as follows:

$$
\begin{aligned}
& a_{1 n}(t)=R \phi_{0}{ }^{2} \omega^{2}[\cos (\omega t)]^{2} \\
& a_{1 t}(t)=-R \phi_{0} \omega^{2} \sin (\omega t)
\end{aligned}
$$

The measurement value of accelerometer $A_{1}$ :

$$
\begin{aligned}
a_{1}(t) & =-a_{1 n}(t) \cos \beta-a_{1 t}(t) \sin \beta \\
& =-\left|O^{\prime} X_{A_{1}}\right| \phi_{0}{ }^{2} \omega^{2} \cos ^{2}(\omega t) \\
& +\left|O^{\prime} Y_{A_{1}}\right| \phi_{0} \omega^{2} \sin (\omega t)
\end{aligned}
$$

The measurement value of accelerometer $A_{2}$ :

$$
\begin{aligned}
a_{2}(t) & =-a_{2 n}(t) \sin \gamma+a_{2 t}(t) \cos \gamma \\
& =-\left|O^{\prime} Y_{A_{2}}\right| \phi_{0}^{2} \omega^{2}[\cos (\omega t)]^{2}-\left|O X_{A_{2}}\right| \phi_{0} \omega^{2} \sin (\omega t)
\end{aligned}
$$

In the solution of the above equation, the measurement values of the accelerometer of $A_{1}$ and $A_{2}$ can be decomposed into the $x$ and $y$ axis of the coordinate system $o_{b}^{\prime} x_{b}^{\prime} y_{b}^{\prime}$, which is equivalent to the sum of the virtual tangential acceleration and the normal acceleration of the coordinate axes. The measurement value of $A_{1}$ is the synthesis of the virtual accelerometer in $X_{A_{1}}$ and $X_{A_{2}}$, and the measurement value of $A_{2}$ is synthesized from the virtual accelerometer in $Y_{A_{1}}$ and $Y_{A_{2}}$.

$Y_{A_{1}}$ is the virtual tangential acceleration:

$$
a_{A_{1} t}(t)=\left|O^{\prime} Y_{A_{1}}\right| \phi_{0} \omega^{2} \sin (\omega t)
$$

$X_{A_{1}}$ is the acceleration of the virtual method:

$$
a_{A, n}(t)=-\left|O^{\prime} X_{A_{1}}\right| \phi_{0}^{2} \omega^{2} \cos ^{2}(\omega t)
$$

$Y_{A_{2}}$ is the virtual tangential acceleration:

$$
a_{A_{2} t}(t)=-\left|O^{\prime} X_{A_{2}}\right| \phi_{0} \omega^{2} \sin (\omega t)
$$

$X_{A_{2}}$ is the acceleration of the virtual method:

$$
a_{A_{2} n}(t)=-\left|O^{\prime} Y_{A_{2}}\right| \phi_{0}^{2} \omega^{2} \cos ^{2}(\omega t)
$$

Refer to the above analysis, the derivation of the virtual tangential acceleration and normal acceleration along the fixed coordinate system of each virtual axial projection, and a point in a cycle of integral sum, we can get the navigation axial velocity increment :

$$
\begin{aligned}
\Delta v_{x}(T) & =\int_{0}^{T} a_{x}(t) d t \\
& =\int_{0}^{T}\left[a_{A 1 n x}(t)+a_{A 1 t x}(t)+a_{A 2 n x}(t)+a_{A 2 t x}(t)\right] d t \\
& =-4\left|X_{A_{1}} X_{A_{2}}\right| \omega f_{1}\left(\phi_{0}, 0, \pi / 2\right) \\
\Delta v_{y}(T) & =\int_{0}^{T} a_{y}(t) d t \\
& =\int_{0}^{T}\left[a_{A 1 n y}(t)+a_{A 1 t y}(t)+a_{A 2 n y}(t)+a_{A 2 t y}(t)\right] \\
& =-4\left|Y_{A_{1}} Y_{A_{2}}\right| \omega f_{1}\left(\phi_{0}, 0, \pi / 2\right)
\end{aligned}
$$

From the above analysis, the navigation speed increment is independent of the rotation center in one period, which is independent of the origin. When the amplitude is fixed, the acceleration of the navigation speed is proportional to the distance from the origin and the distance between the axial velocity and the rotation angular velocity ${ }^{[5]}$. In practice, if the accelerometer is used as A point measurement component, the navigation error will be generated from the principle, and the calculation error will be formed. We will call that $r_{1}=\left|X_{A_{1}} X_{A_{2}}\right| 、 r_{1}=\left|Y_{A_{1}} Y_{A_{2}}\right|$ is the inner arm length.

Using vector analysis method: if there is no internal lever error, then accelerometer of $P$ point:

$$
a_{P}=a_{0}
$$

In fact, if there is an internal lever error, the acceleration at $\mathrm{P}$ point can be obtained as:

$$
a_{P}=a_{0}+\dot{\omega} \times r_{P}+\omega \times\left(\omega \times r_{P}\right)
$$

$\omega \times r_{P}$ and $\omega \times\left(\omega \times r_{P}\right)$ respectively represent the tangent acceleration and normal acceleration of $\mathrm{P}$ points relative to $O_{b}$ points.

The inner rod effect refers to the centripetal force and tangential force that the accelerometer detected during the movement of the carrier due to the relative ideal location $^{[6]}$.

As a result of the three accelerometer sensitive shaft extension cord to the intersection point, so the lever arm within error of accelerometer is introduced by $\omega \times\left(\omega \times r_{P}\right)$. Assume that the accelerometer's sensitive axes coincide with the vector coordinate system, due to the sensitive sensitive accelerometer only along the axis direction of the acceleration, the projection on the axis of accelerometer lever arm in the corresponding error can be represented as:

$$
\begin{aligned}
& a_{e}=\left[\begin{array}{lll}
a_{e x} & a_{e y} & a_{e z}
\end{array}\right]^{T} \\
& \left\{\begin{array}{l}
a_{e x}=-\left(\omega_{y}^{2}+\omega_{s}^{2}\right) r_{x} \\
a_{e y}=-\left(\omega_{x}^{2}+\omega_{s}^{2}\right) r_{y} \\
a_{e z}=-\left(\omega_{y}^{2}+\omega_{x}^{2}\right) r_{s}
\end{array}\right.
\end{aligned}
$$

In particular, when there are oscillatory motion, the oscillatory motion passes through the rectifier, it will 
produce a constant acceleration error.

when $\omega_{y}=\omega \theta_{y} \sin \omega t, \quad \omega_{s}=\omega \theta_{s} \sin (\omega \mathrm{t}+\varphi)$ :

$$
\begin{aligned}
a_{e x} & =-\left\{\omega^{2} \theta_{y}^{2} \sin ^{2} \omega t+\omega^{2} \theta_{s}^{2} \sin ^{2}(\omega t+\varphi)\right\} r_{x} \\
& =-1 / 2 \omega^{2}\left(\theta_{y}^{2}+\theta_{s}^{2}\right) r_{x} \\
& +1 / 2 \omega^{2}\left\{\theta_{y}^{2} \cos 2 \omega t+\theta_{s}^{2} \cos (2 \omega t+2 \varphi)\right\} r_{x}
\end{aligned}
$$

The upper formula indicates that the size effect causes a constant acceleration zero deviation, which is $\omega^{2}\left(\theta_{y}^{2}+\theta_{s}^{2}\right) r / 2$. And when the amplitude of the periodic motion is 0.1 , and the frequency is $50 \mathrm{HZ}$. The position deviation of $10 \mathrm{~cm}$ will produce about $1.5 \mathrm{mg}$ of the acceleration zero deviation. Therefore, the error of the accelerometer size effect will not be avoided in the process of finding the north.

The measurement output of the accelerometer can be expressed as:

$$
a_{P}=a_{0}-a_{e}=\left[\begin{array}{c}
a 0 x \\
a 0 y \\
a 0 z
\end{array}\right]-\left[\begin{array}{c}
\left(\omega_{y}^{2}+\omega_{s}^{2}\right) r_{x} \\
\left(\omega_{x}^{2}+\omega_{s}^{2}\right) r_{y} \\
\left(\omega_{y}^{2}+\omega_{x}^{2}\right) r_{s}
\end{array}\right]
$$

The form of vector notation is:

$$
a_{0}=a_{P}-\omega \times\left(\omega \times r_{P}\right)
$$

The upper equation is the compensation form for the error of the inner rod arm. If the $\mathrm{O}$ point is viewed as the output point of the accelerometer, the inner rod arm error will not exist.And the measurement result of the addition will not be affected by the principle.

When the three accelerometer sensitive shaft extension cord are not intersect in one point, the size effect error will inevitably related to the value of the derivative of angular velocity, which can cause the tangential acceleration. This article does not consider this situation. This requirement can be achieved in the current assembly process. Therefore, this study is not studied in this paper.

\section{The error of inner rod is affected by single axis rotation}

Based on the single-axis IMU turntable in the laboratory, the accuracy of the error modeling and the validity of the error compensation are verified in this paper. The effect of the inner rod effect on the north precision of the system is analyzed.

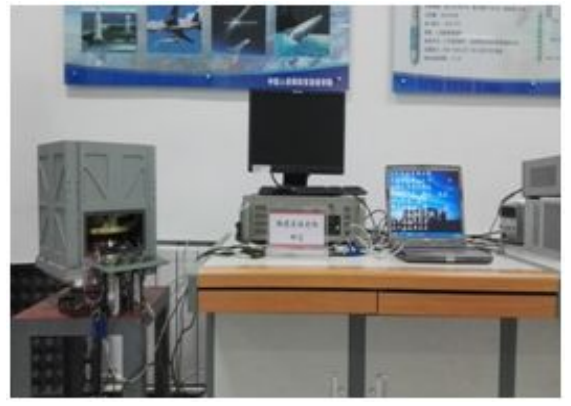

Figure 3. Experimental setup diagram

The test process is as follows: (1) Initial alignment of work mode: divided into coarse alignment and precise alignment process. Using the method of document 5, we have added the compensation scheme for the error of the inner rod arm in the scheme. (2) The autonomous inertial navigation mode: collect the output data of IMU for navigation in the navigation computer, analyzing the validity of error compensation scheme after modulation results. The experiment principle as shown in figure 4 .

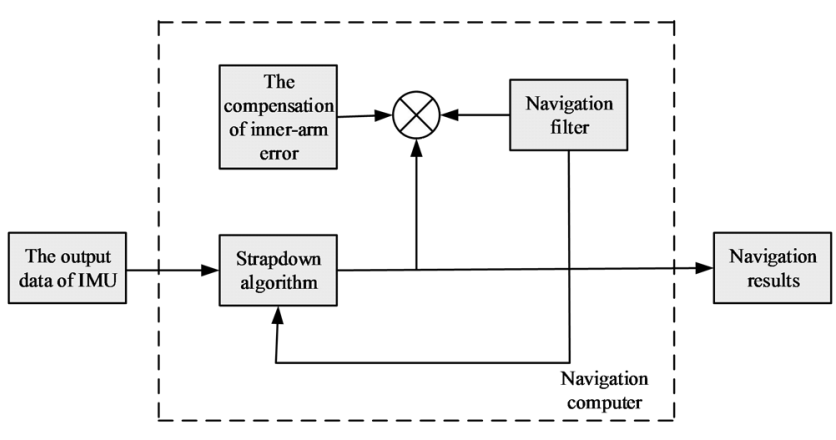

Figure 4. The principle block diagram of the SINS navigation system

During the experiment, the rotary table is guaranteed to be in constant position, position and orientation, and conduct multiple navigation experiments. By error analysis and data processing of the results of the navigation settlement, the error suppression is carried out by using kalman filter algorithm, and the results are shown as:

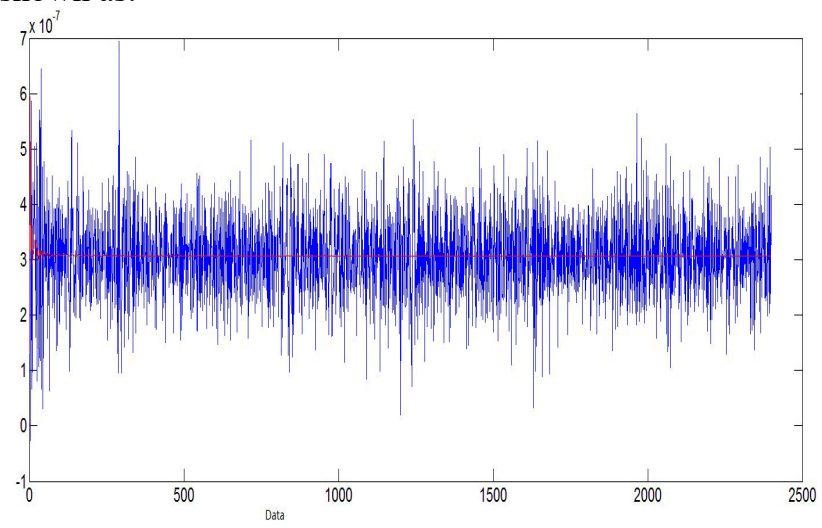

Figure 5. Filter simulation results (Blue-the original data; Red-the post-filtered data)

After the rod arm error compensation, it can be seen 
from fig. 5 that the error of output data is significantly reduced. In the process of uniaxial rotating find north, the existence of the rotating device will increase the size effect of error, this is equivalent to the introduction of the new constant drift, cause acceleration got an additional acceleration signal measuring. During the long rotation process, the existence of the rotating device can realize the offset of the error, which will not lead to the divergence of navigation positioning error. The theoretical analysis and simulation results show that the size error of the accelerometer will not lead to the divergence of positioning error, but it will increase the oscillation amplitude of the undamped system speed error.

\section{Conclusion}

In this paper, under the condition of the accelerometer perpendicular installation, detailed in the principle of lever arm effect error formula was deduced and analyzed the causes, the analysis results: navigation speed increment within lever-arm effect has nothing to do with the center of rotation, and is proportional to the inside lever arm length, and is proportional to the IMU rotation angular velocity. The influence of the error effect of the inner rod arm in the process of the homing system is analyzed. This article only for vertical installation situation carries on the analysis and discussion, if three accelerometer is not vertical or sensitive shaft extension cord is not intersect in one point, in this case the error analysis and compensation discussion is more complex, needed further in-depth analysis.

\section{References:}

1. Yuan Bao-lun, Rao Gu-yin.On the theory of Optical Gyro Rotating Inertial navigation System[J].Journal of National University of Defense Technology, 2007,28(6):76-80.

2. David H Titterton, John L Weston.Strapdown Inertial Navigation Technology[M]. Zhang Tian-guang, Wang Xiu-ping,et al.BeiJing: National Defense in Dustry Press, 2007.

3. Yan Gong-min, Yan Wei-sheng, Xu De-min. Analysis and Compensation on Inner Lever Arm Effect of Strapdown Inertial Measurement Unit[J]. Journal of Chinese Inertial Technology, 2008, 16(2):148-153.

4. Chen Yong-bing, Zhong Bin.Inertial Navigation Principle[M].Bei Jing:National Defense in Dustry Press, 2007.

5. Yu Xu-dong,Wang Yu,Zhang Peng-fei,et.Influence of Single-axial Rotation on INS Error Characteristics[J].Journal of Chinese Inertial Technology, 2008,16(6):643-648.

6. Huang Xi-cheng,Research of the High Precision
North Finding Technology Using Ring Laser Gyroscope[D] :Harbin Institute of Technology, 2013,09 . 